\title{
DESIGN OF A HIGH-PRECISION TUNABLE DOUBLE CRYSTAL MONOCHROMATOR
}

M. Shleifer, S. Sharma, E. Rotela, C. Brite

February 1992

\section{NATIONAL SYNCHROTRON LIGHT SQURCE}

\author{
BROOKHAVEN NATIONAL LABORATORY \\ ASSOCIATED UNIVERSITIES. INC

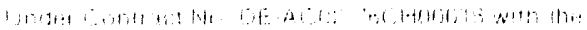 \\ UNITED STATES DEPARTMENT OF ENERGY

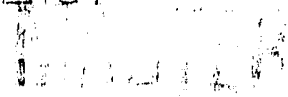

DETRELLTION OF THIS DOCUMENT IS UNLIMITEO 
$1.1 .41,21111 \cdot 1:$

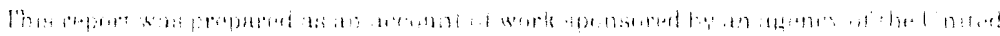

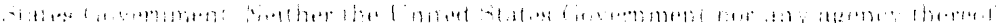

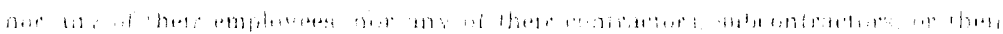

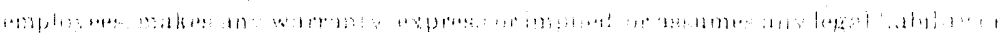

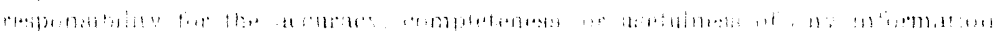

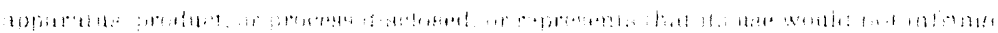

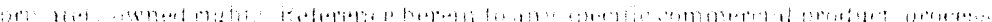

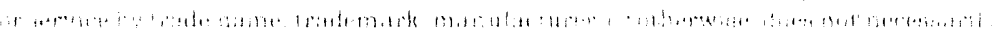

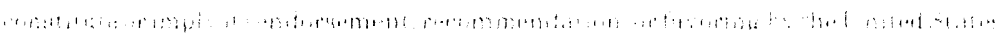

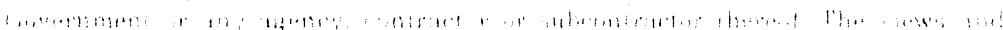

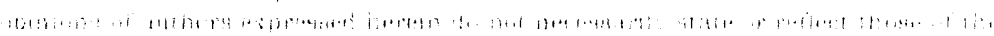

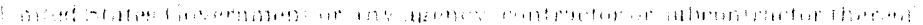




\title{
DESIGN OF A HIGH-PRECISION TUNABLE DOUBLE CRYSTAL MONOCHROMATOR*
}

\author{
BNL --47194
}

\author{
M. Shleifer, S. Sharma, E. Rotela, C. Brite \\ National Synchrotron Light Source \\ Brookhaven National Laboratory \\ Upton, New York 11973
}

DE92 010137

\begin{abstract}
The design of a high-precision tunable doubie crystal monochromator is described. Each crystal of the monochromator can be aligned independently by a double-axis gimbal mechanism providing rotations about two perpendicular axes. Two orthogonal linear actuators operate the gimbal mechanism via a connecting bar. The exit beam height is controlled by separate linear actuators that change the distance becween the crystals along the beam axis. Vertical separation between the crystals may also be acjusted. Thermal deformations are minimized in this compact UHV compatible design by water cooling of the first crystal.
\end{abstract}

\section{INTRODUCTION}

The design of double crystal monochromators has received considerable attention in receni years. Various monochromator designs have been developed [1-5] to meet many of the stringent design requirements for specific experiments. The design requirements include: (1) rapid tunability with fixed exit beam position, (2) good energy resolution and repeatability, (3) cooling of the first crystal to remove the absorbed power, and (4) remote operation. In addition, the monochromators are required to be UHV compatible in many applications.

In this report we propose a compact design for a high-precision double crystal monochromator that satisfies the above design requirements. The design employs a double-axis gimbal mechanism to provide rotations with high angular resolution. The gimbal mechanism has been used previously in the design for a UHV mirror box [6] that has been in successful operation on the U13 beamline at the NSLS.

*Work performed under the auspices of U.S. DOE under contract DE-ACO2-76CH00016. 


\section{DESIGN CRITERIA}

In orue to keep the design simple and compact without sacrificing high precision, the following design criteria were established:

(1) A digital feedback system should be used for aligning the two crystals instead of mechanical linkages which tend to be long and imprecise for small Bragg angles. Mechanical linkages are also inconvenient if closed feedback systems are employed to cancel the exit beam movements due to closed orbit distortions of the beam in the storage ring [7].

(2) Crystal rotations about two perpendicular axes should be accurate to within a fraction of an arcsecond. Our previous experience has shown that this can be accomplished with a simple inexpensive double-axes gimbal mechanism manipulated by linear actuators via a long connecting bar.

(3) The exit bear position should be maintained within one micron. The exit beam position can be controlled either by changing the distance (measured along the beam axis), or the gap (measured perpendicular to the crystal plane) between the crystals. For a change of one micron in the distance or gap between the crystals, the beam position shifts by $\sin 2 \theta_{9}$ and $2 \cos \theta_{\mathrm{B}}$, respectively, where $\theta_{\mathrm{B}}$ is the Bragg angle. The first approach has been adorted in the present design since it privides higher resolution for adjusting the beam position.

(4) For easy installation and maintenance, the drive mechanism for each crystal should be assembled as a removable spindle on a conflat flange.

(5) The entire mechanism should be UHV compatible and should provide for watercocling of the first crystal.

\section{MECHANICAL DESIGN}

Figure 1 shows the general layout of the monochromator consisting of a housing (1), a crystal spindle (2) with provisions for water cooling, and another crystal spindle (3) that does not. require water cooling.

Each crystal spindle (see Fig. 2) is mounted on a conflat flange (4) which is rigidly connected to the top part of a horizontal slide (5). The bottom part of this slide is attached to a sub-assembly (6) consisting of a frame (7) mounted on four shafts and two end plates as shown in the figure. Vertical motion of the frame is guided along the four shafts via eight bushings (8). A stepper motor (9) controls the vertical motion through a gear reducer (10) and a screw (11). The screw transmits the motion to a rigid tube (12) attached to a base plate (13) that supports the double gimbal mechanism. An intermediate cross-plate (14) is movnted on the base plate via a set of bearings (15) shown in the top view. A bottom plate (16) is mounted on the cross-plate by another set of identical bearings with their axis perpendicular to the first set. A water-cooled 
molybdenum block (17) supporting the monochromator crystal (18) is fixed to this bottom plate. A special liquid indium-gallium eutectic alloy interface is used between the molybdenum block and the crystal in order to reduce the interface thermal resistance.

The crystal and the cooling block are thus attached to the bottom plate mounted on two sets of bearings which can be easily rotated about two perpendicular axes. Rotations are provided by two linear actuators (19) and (20) through a connecting bar (21). One end of this bar is connected to the slides via a bushing (22) and a spherical cap (23), and the second end is fixed to the bottom plate (16). A set of two bellows, (24) and (25), are used to maintain the UHV compatibility of this mechanism under various relative motions. The bellows (24) permit angular motions of the connecting bar, whereas the bellows (25) allow for vertical and lateral movements of the spindle. Two tubes (26) for water-cooling of the molybdenum block are installed inside the connecting bar (21) for the first crystal spindle only.

For the design dimensions shown in Fig. 2, the Bragg angle can be changed by $\pm 3^{\circ}$ from its nominal position. The bellows (25) allow an adjustment of $\pm 50 \mathrm{~mm}$ in the distance between the two crystals. This range of adjustment is required to offset the change in the exit beam position when the two crystal are vertically separated by $10-15 \mathrm{~mm}$ as in the present design. Adjustments to the Bragg angle and the distance between the two crystals are made under remote computer control. Fine adjustments are made by a digital feedback system receiving. signals from a photon beam position monitor placed in the path of the exit beam. The same feedback system may be used to cancel the time dependent shift in the exit beam position due to closed orbit deviations of the beam in the storage ring [7].

The tunability range can be increased by combining two monochromators set at different nominal Bragg angles. For instance, the first monochromator can be set at a nominal Bragg angle of 8.5 degrees, covering a range of $10-20 \mathrm{keV}$ ( $\mathrm{Si}<111>$ crystals), whereas the second monochromator can cover the higher energy range of $20-100 \mathrm{keV}$ when set at a nominal Bragg angle of 3 degrees. Similarly, for $\mathrm{Si}<220\rangle$ crystals the two monochromators can be set to cover energy ranges of $15-25 \mathrm{keV}$, and greater than $25 \mathrm{keV}$, respectively. When using the second monochromator, the first crystal of the first monochromator is retracted using the vertical motion of the frame ( 7$)$ in order to clear the incident beam.

A high precision in setting the Bragg angle is achieved in this monochromator design because angular rotations are converted into comparatively large linear translations via the long connecting bar (21). An absolute accuracy of 0.5 arcsecond and a resolution of 0.05 aresecond can, for example, be obtained using a $400 \mathrm{~mm}$ long connecting bar with a linear actuator that has an absolute accuracy and a resolution of 1 micron and 0.1 micron, respectively (e.g. Klinger linear actuator, Model VP30). 


\section{REFERENCES}

[1] P. L. Cowan, J. B. Hastings, T. Jach and J. P. Kirkland, Nucl. Instr. and Meth. 208 (1983) 349.

[2] D. M. Mills and M. T. King, Nucl. Instr. and Meth. 208 (1983) 341.

[3] T. Ohta, P. M. Stefan, M. Nomura and H. Sekiyama, Nucl. Instr. and Meth. A246 (1986) 373.

[4] H. Kawata and M. Ando, Nucl. Instr. and Meth. A246 (1986) 368.

[5] S. M. Heald, Nucl. Instr. and Meth. A266 (1988) 457.

[6] S. L. Hulbert, E. Rotela and M. Shleifer, Nucl. Instr. and Meth. A291 (1990) 337.

[7] A. Koyama, S. Sasaki and T. Ishikawa, Rev. Sci. Instrum. Vol. 60, No. 7, (1989) 1953. 


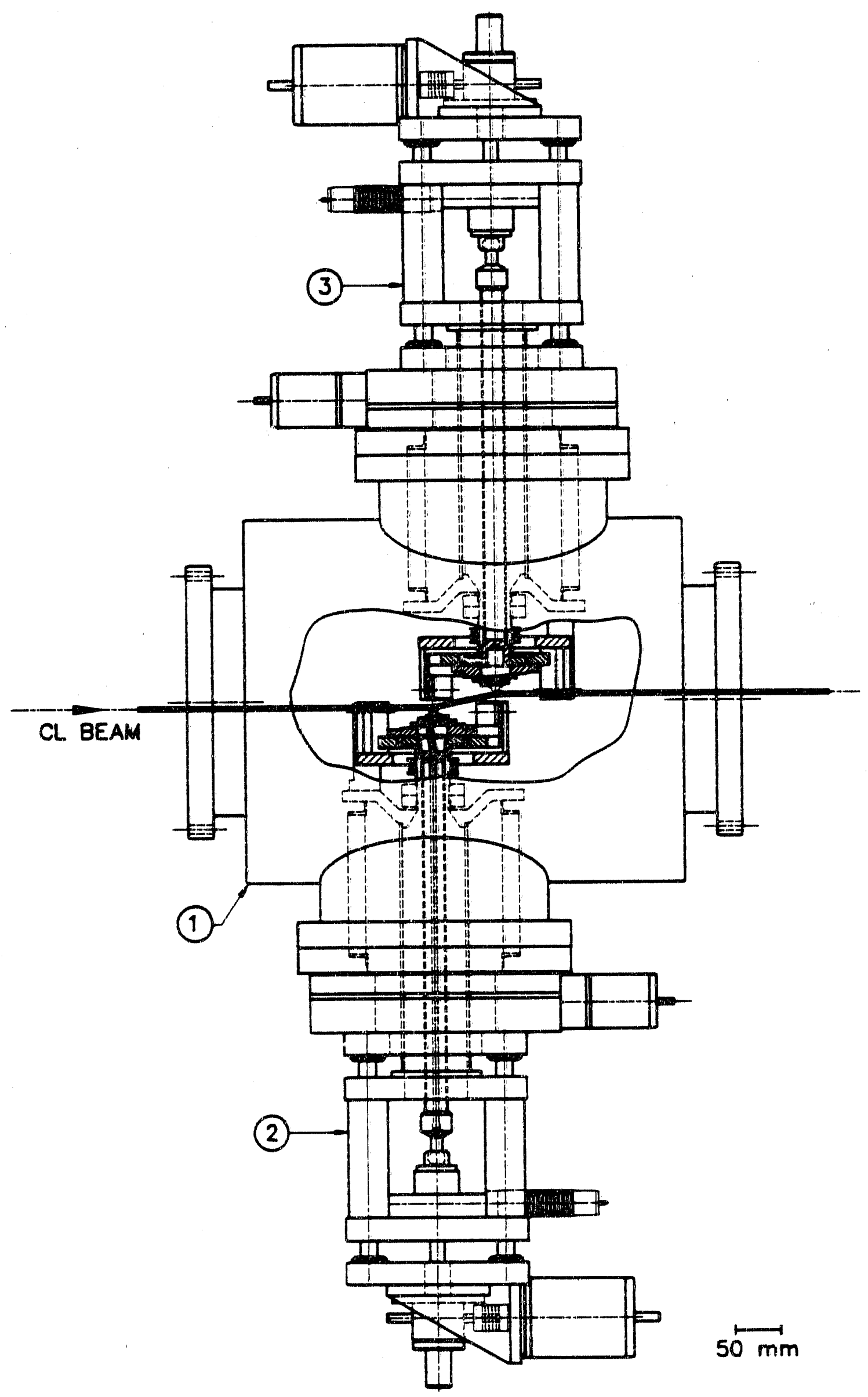

FIC. 1 A TUNABLE DOUBLE CRYSTAL MONOCHROMATOR 


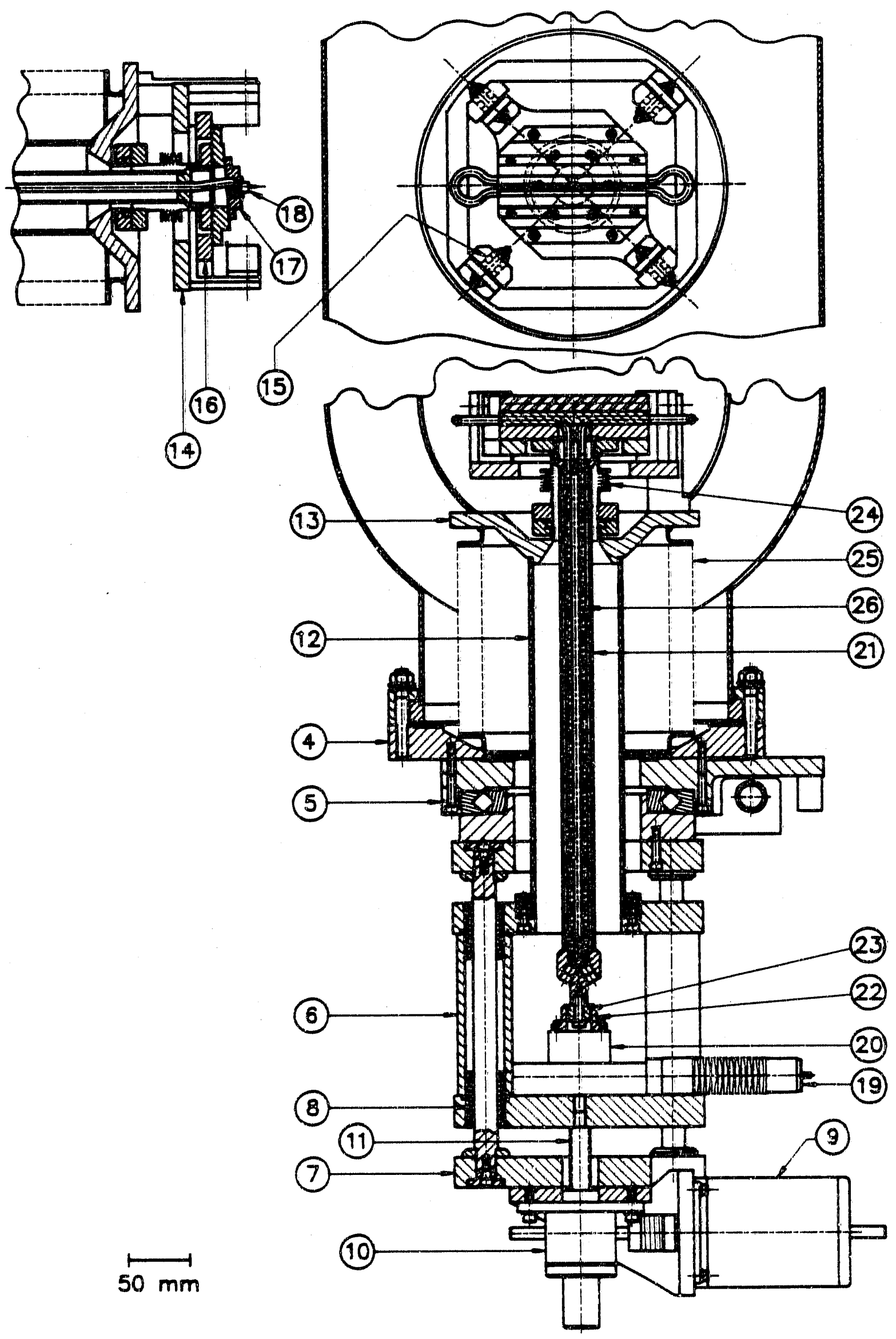

FIG. 2 WATER-COOLED SPINDLE FOR THE FIRST CRYSTAL 

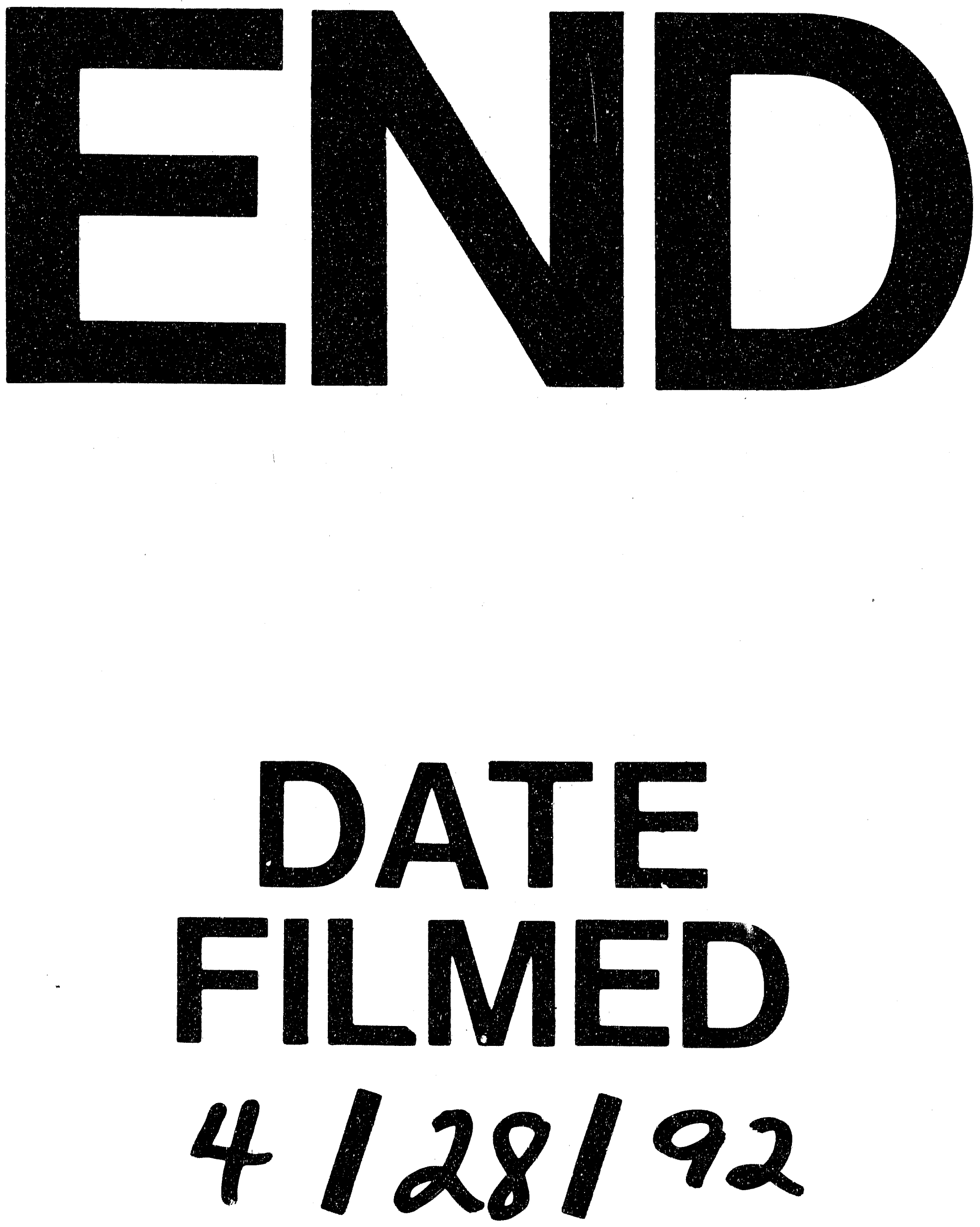
